\title{
Diagnostische Bedeutung des Prolaktinspiegels in der Gynäkologie
}

H. Giesel und L Mettler

Abteilung Frauenheilkunde im Zentrum Operative Medizin I der Christian-Albrechts-Universität Kiel und Staatiche Hebammenlehranstalt (Direktor: Prof. Dr. K. Semm)

\section{Zusammenfassung:}

Der spezifische radioimmunologische Prolaktinnachweis gelang erstmalig 1971. Die Regulation der Prolaktinsekretion erfolgt über hypothalamische Neurrotransmitter, die eine hemmende Wirkung auf die Prolaktin-produzierenden chromophoben Zellen des Hypophysenvorderlappens ausüben. Die Zufuhr von TRH oder Östrogenen verursacht eine erhöhte Prolaktinfreisetzung.

Der Prolaktin-Serumspiegel ist vom Schlaf-Wach-Rhythmus abhängig. Physiologisch efhöhte Werte finden sich während der Schwangerschaft und Stillzeit bei der Mutter sowie im Fruchtwasser und beim Feten.

Die medikamentöse Hemmung dopaminerger Prozesse führt zu einem Anstieg, die Gabe von Bromokryptin zu einem Abfall des Prolaktinspiegels.

Beim ,hyperprolaktinämisch-anovulatorischen Syndrom" mit oder ohne Galaktorrhoe muß ein Hypophysen-Adenom als Ursache ausgeschlossen werden.

Entsprechend seiner stammesgeschichtlichen Bedeutung werden dem Prolaktin auch beim Menschen noch vielfältige, bisher nicht bestätigte Einflüsse zugeschrieben.

\section{Schlüsse/wörter:}

Prolaktin, Reproduktion - hyperprolaktinämisch-anovulatorisches Syndrom - Hypophyse - Bromokryptin.

Summary:

The regulation of the only in 1971 specified hormon prolactin is done by hypothalamic neurotransmitter substances, which inhibit prolactin-producing cells in the anterior hypophysis. With TRH and estrogens sufficient prolactin secretion is produced.

Alterations in human prolactin serum levels result through a dependency on sleep-and awake-rhythms.

Physiological increased values are detected during pregnancy and parturition as well as in the fetus and its surrounding amniotic fluid.

The medicamental inhibition of dopaminergic processes leads to an increased prolactin secretion. A secure suppression is achieved with bromocryptin.

As causal factor of the "hyperprolactinaemic-anovulatory syndrom" with or without galactorrhoea a tumor of the hypophysis has to be excluded.

Corresponding to the great developmental significance of prolactin also in the human a series of other effects are suspected. This has, however, not yet been verified.

Key words:

Prolactin - Reproduction - Hyperprolactinaemic-anovulatory syndrom - Hypophysis Bromocryptin. 


\section{Einleitung}

Prolaktin ist ein Polypeptid-Hormon aus den chromophoben Zellen des Hypophysenvorderlappens. Es besteht aus 198 Aminosäuren und besitzt ein Molekulargewicht von etwa 20000 . Bereits in den dreißiger Jahren gelang die erstmalige Isolierung aus tierischem Eiweiß, aber erst 1971 konnte die Existenz des Prolaktins als selbständiges Hormon neben dem in seiner Aminosäurensequenz sehr ähnlichen Wachstumshormon $(\mathrm{GH})$ bewiesen werden (11).

Die geringe Empfindlichkeit und die Unspezifität der ersten Prolaktinnachweise wurde abgelöst durch spezifische radioimmunologische Methoden zur quantitativen Bestimmung des Hormons beim Menschen (9). Seither ist das Wissen um die klinische Bedeutung des Prolaktins außerordentlich rasch gewachsen, nicht zuletzt durch die Möglichkeit, die Prolaktinsekretion durch Pharmaka gezielt zu beeinflussen (5).

\section{Steuerung der Prolaktinsekretion}

Beim Menschen erfolgt die Regulation der Prolaktinsekretion hauptsächlich durch Hemmung der Prolaktinproduzierenden Zellen des Hypophysenvorderlappens. Die Unterbindung dieser inhibitorischen Steuerung durch Hypophysenstiel-Durchtrennung führt jedenfalls zu einer eindrucksvoll erhöhten Prolaktinsekretion (6).

Bei der als Prolactin Inhibiting Factor (PIF) bezeichneten Substanz handelt es sich um einen dopaminergen Neurotransmitter, der aus hypothalamischen Extrakten gewonnen werden kann. Daneben vermutet man die Existenz eines Prolactin Releasing Factor (PRF) als weiteren hypothalamischen Steuerungsfaktor. Gesichert ist dagegen, daß der Thyreotropin Releasing Factor (TRH) die hypophysäre Prolaktinfreisetzung zu stimulieren vermag. Wegen seiner prompten Wirkung kann er zur Beurteilung der "Prolaktinreserve“ der Hypophyse angewendet werden. PIF und TRH bzw. auch PRF gelangen über das hypophysäre Pfortadersystem in den Hypophysenvorderlappen und bewirken hier eine Hemmung bzw. Freisetzung des Prolaktins.

Die Normalwerte der Prolaktin-Serumkonzentrationen liegen bei beiden Geschlechtern zwischen $5-25 \mathrm{ng} / \mathrm{ml}$. Ein typisches Sekretionsmuster im Menstruationszyklus, wie für Gonadotropine und Sexualsteroide, gibt es nicht. Ähnlichkeit mit anderen Proteohormonen der Hypophyse zeigen sich jedoch bei den echten Kurzzeitschwankungen der Prolaktinsekretion. Leighton et al. (11) (1975) beobachteten pulsatile Hormonfreisetzung aus der Hypophyse, wobei das zeitliche Intervall 1-2 Minuten beträgt.

Der aktuelle Prolaktin-Serumspiegel ist ferner abhängig vom Schlaf-Wach-Rhythmus. 1-2 Stunden nach Beendigung des Schlafs haben sich üblicherweise die Prolaktinwerte wieder auf das Tagesnieveau eingepen- delt (2). Diese Beobachtungen machen deutlich, wie wichtig der Zeitpunkt der Blutabnahme für die Beurteilung des Einzelwertes ist.

Aufgrund der höheren Östrogenproduktion während der Geschlechtsreife liegen bei der Frau die ProlaktinSerumspiegel etwas höher als beim Mann (17). Der Wirkungsmechanismus, über den Östrogene und auch Progesterone einen Stimulus für die Prolaktinfreisetzung darstellen, bedarf noch der weiteren Abklärung. Schon eine geringe Östrogen-Dauermedikation - wie unter der hormonalen Kontrazeption - führt zu einer signifikanten Steigerung der Basalsekretion (17).

\section{Physiologische Hyperprolaktinämie}

Prolaktin wird unter verschiedenen physiologischen Bedingungen irn Serum erhöht gefunden (Tab. 1). Diese Konzentrationszunahme ist nicht etwa. Folge einer verminderten Hormonclearance, sondern Folge vermehrter Sekretion. Verantwortlich dafür sind sowohl zentral-neurale Vorgänge sowie sensorische Afferenzen und Stoffwechselveränderungen. So finden sich physiologisch erhöhte Prolaktinwerte außer in Schlafphasen auch nach körperlichen Anstrengungen und operativen Eingriffen sowie infolge bestimmter Streßsituationen.

Schwangerschaft und Wochenbett stellen ebenfalls Phasen physiologisch erhöhter Prolaktinämien dar. Im mütterlichen Serum steigt der Prolaktinspiegel ab etwa der 5.-7. Schwangerschaftswoche kontinuierlich bis zum Termin um ca. das 10-fache an, wobei eine auffallende Parallelität im Verhalten der Serumkonzentrationen von Prolaktin und Ostradiol besteht (1).

Nach der Geburt fallen die mütterlichen Prolaktinwerte relativ langsam ab. Die Prolaktinsekretion im Wochen-

Tab. 1:

Physiologische. Zustände erhöhter Prolaktinfreisetzung

\section{Schlaf}

Körperliche Anstrengung

Streß

Schwangerschaft

Wochenbett

Reizung der Brustwarzen

Koitus

Prä- und Perinatalphase des Kindes 
Abb. 1:

Klassifizierung der Ovarialinsuffizienz

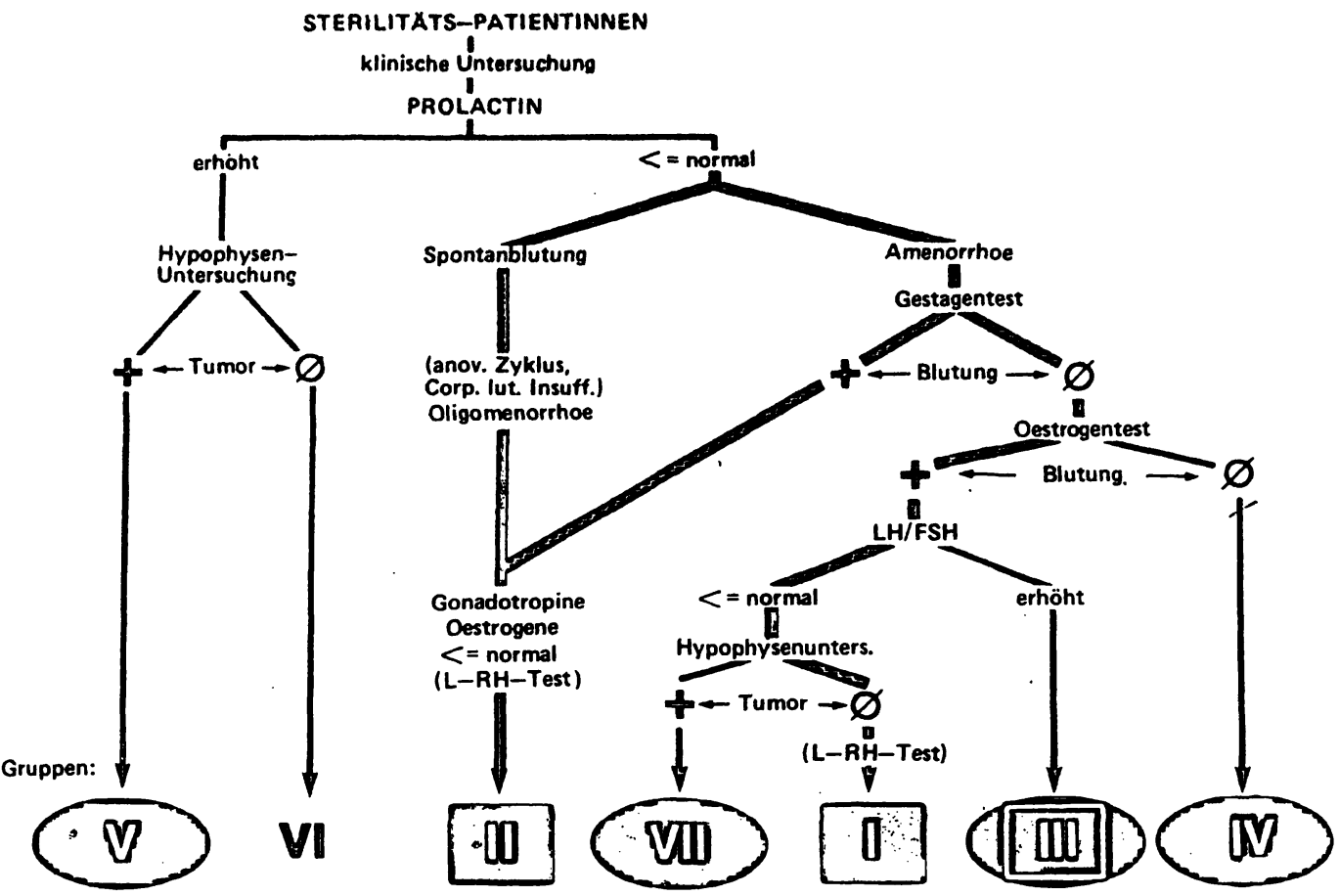

bett zeichnet sich vor allem durch die Stimulierbarkeit im Zusammenhang mit dem Stillen aus. Danach führt das Saugen des Kindes an der Brustwarze der Mutter innerhalb von 10 Minuten zu einem Anstieg des Prolaktinspiegels im mütterlichen Serum. Nach Absetzen des Kindes fallen die Spiegel rasch ab, so daß 3 Stunden später die Ausgangswerte wieder erreicht sind.

Wie bei der Mutter, so kommt es auch beim Feten mit zunehmendem Gestationsalter zu einem Anstieg der Prolaktin-Serumkonzentration.

Von allen untersuchten Körperflüssigkeiten enthält das Fruchtwasser die höchsten Prolaktin-Konzentrationen mit Spitzenwerten zwischen der 22. und 26. Schwangerschaftswoche.

\section{Medikamentöse Beeinflussung der Prolaktinsekretion}

Eine Verringerung der Prolaktinausschüttung kann durch einen orale Wasserbelastung bzw. intravenöse hypotone Volumenexpansion ausgelöst werden (3). Daneben können einige Pharmaka zu einer Hemmung der Prolaktinsekretion führen (Tab. 2). Neben dem Anti-Parkinson-Mittel L-Dopa - es handelt sich um eine metabolische Vorstufe für den Neurotransmitter Dopamin - und dem spezifischen Stimulator der Dopaminrezeptoren, dem Apomorphin, das mehr von experimenteller Bedeutung ist, ist das Mutterkorn AlkaloidDerivat Alpha-bromo-ergo-Kryptin (Pravidel ${ }^{\circledR}$, Sandoz) der wichtigste Vertreter der die Prolaktin-Produktion hemmenden Substanzen. Alle genannten Stoffe können als Dopaminrezeptoren anregend charakterisiert werden (6).

Eine Reihe häufig verwendeter Psychopharmaka - hier besonders die Phenothiacine, aber auch Reserpin sowie Alpha-Methyldopa-stimulieren die Prolaktinsekretion. Als gemeinsame Eigenart dieser Klasse läßt sich die Hemmung dopaminerger Prozesse herauskristallisieren. Auf der anderen Seite gibt es aber auch Stoffe wie das Thyreotropin Releasing Hormon (TRH), wélches nicht nur eine TSH-Ausschüttung bewirkt, sondern sich als 
das wirkungsvollste Stimulans der Prolaktinsekretion erwiesen hat. Der Einfluß von Östrogen und auch Progesteron auf die Prolaktin-Produktion sei hier noch einmal der Vollständigkeit halber erwähnt.

\section{Pathologische Hyperprolaktinämien}

Bei etwa 20\% aller amenorrhoeischen Frauen muß nach Ausschluß anlagebedingter und anatomischer Veränderungen des Genitale eine Hyperprolaktinämie als Ursache der Amenorrhoe angenommen werden.

Das Krankheitsbild des „Hyperprolaktinämisch-anovulatorischen Syndroms" stellt somit eine der häufigsten Ursachen der anovulatorischen Sterilität dar (18). Dem trägt die WHO-Klassifizierung $(21,14)$ zur endokrinen Diagnostik der Sterilität Rechnung, in der die Hyperprolaktinämie als Leitsymptom aufgeführt wird (Abbildung 1). Nach Ausschluß eines Hypophysentumors (Gruppe V) erfolgt die Therapie in der Gruppe VI mit dem AntiProlaktin-Bromoergo-Kryptin (15).

Bei 10-20\% aller Frauen mit sekundärer Amenorrhoe läßt sich auch eine Hyperprolaktinämie nachweisen, wenn keine Galaktorrhoe besteht (19).

Bei den klinischen Symptomen wie Amenorrhoe, Anovulation und bis zu einem gewissen Grad die Corpus luteum-Insuffizienz wird nicht selten ein Hypophysentumor als Ursache für eine Hyperprolaktinämie gefunden. Es ist daher angebracht, bei jeder form der Hyperprolaktinämie, mit oder ohne Galaktorrhoe, ein intra- oder supraselläres Tumorgeschehen auszuschließen.

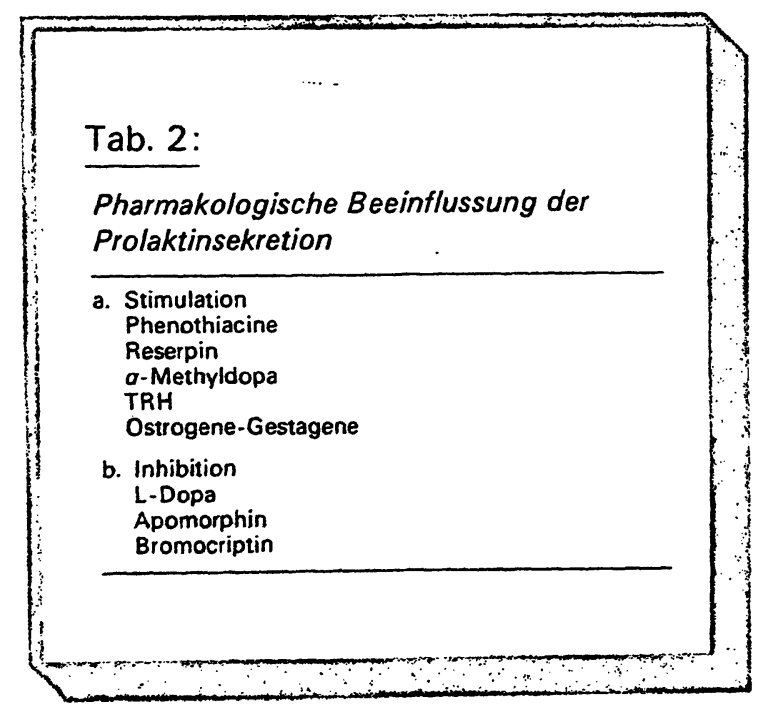

Es handelt sich vorwiegend um chromophobe Hypophysentumoren. Im Prinzip kann jeder intracranielle Prozeß zu einer Beeinträchtigung der hypothalamischen-hypophysären Kommunikation führen. Dadurch wird der hemmende Einfluß des PIF vermindert oder aufgehoben, und es kommt zur gesteigerten Prolaktinsekretion (Tab: 3).

Aus diesen Gründen müssen sorgfältige neuro-radiologische sowie opthalmologische Untersuchungen, insbesondere Schichtaufnahmen der Sella, computertomographische Unterșuchungen sowie Sehschärfenund Gesichtsfeldbeśtimmungen durchgeführt werden, um auch kleinste Veränderungen, wie z. B. Doppelkonturen des Sellabodens, auszuschließen.

Bei Vorliegen eines erhöhten Prolaktinspiegels im Serum muß solange ein Adenom oder Mikroadenom der Hypophyse angenommen werden, bis das Gegenteil bewiesen werden kann (10).

Eine ausgeprägte Hypothyreose kann auf der Basis von Prolaktinfreisetzung durch TRH zu einer Hyperprolaktinämie führen. Schilddrüsenfunktionstests sind daher ebenfalls ein notwendiger Bestandteil zur Abklärung einer Hyperprolaktinämie.

Weitere seltenere Ursachen eines erhöhten SerumProlaktinspiegels umfassen die ektopische Hormonproduktion durch maligne Tumoren sowie Nierenversagen, wahrscheinlich durch die reduzierte Nierenclearance des Prolaktins.

Die einzige Hypophysenerkrankung, die gewöhnlich ein erniedrigtes Prolaktin zur Folge hat, ist das SHEEHAN-Syndrom.

Vielfältige Theorien über weitere Rollen des Prolaktins

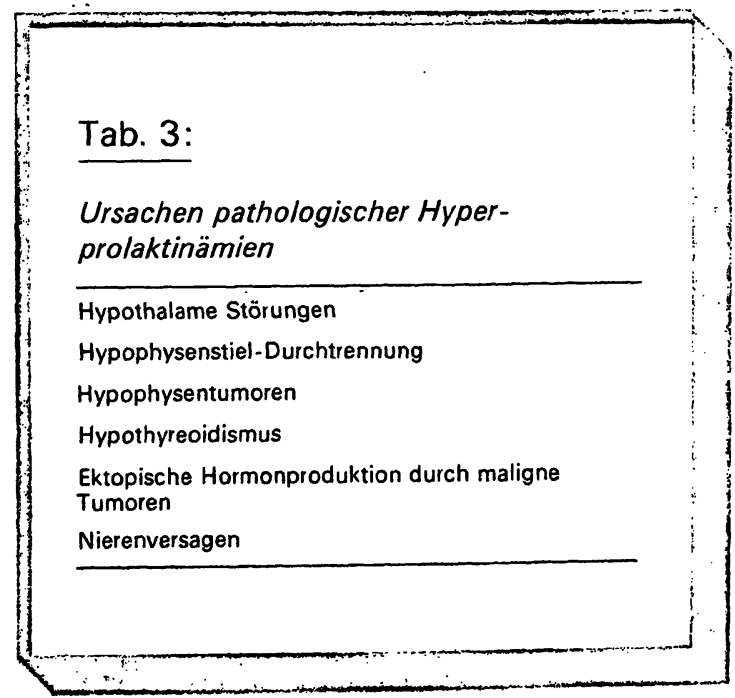




\section{im Körperstoffwechsel bedürfen noch ihrer endgültigen Bestätigung. So ist die Bedeutung des Prolaktins beider Entstehung und Therapieuberwachung des Mamma- Carcinoms unstritten (4).}

Prämenstruelles Syndrom. Migräne (7) sowie Präeklampsie (8) werden ebenfalls mit dem weiten Wirkungsspektrum des Prolaktins in Verbindung gebracht. Jedenfalls ließ sich durch Gabe von Bromokryptin bei Mastodynie und anderen Symptomen des prämenstruellen Syndroms und bei Frauen mit zyklusabhängigen Migräneattacken eine deutliche Besserung des Beschwerdebildes erzielen, auch wenn keine Hyperprolaktinämie nachgewiesen werden konnte (20).

Anschrift der Verfasser:

Dr. med. H. Giesel

Priv.-Doz. Dr. med. L. Mettler

Universitäts-Frauenklinik Kiel

Hegewischstr. 4

D-2300 Kiel 1

\section{Schoriftum}

1. BELLMANN. O. Prolaktin in der Schwangerechatt und im Wochentett - zu Sekretion Regulation und Funktion. Gynakologe 10,66 (1977)

2 BOYAR. R. M FINKELSTEIN. J. W.. KAPEN. S., HELLMANN. L.: Twonty-four hour prolectin (PRL) excretory pattorns during pregnancy. S. clin. Endoct. 40, 1117 (1975)

3. BUCKMANN, M. T., KAMINSKY, N., CONWAY, M., PEAKE, G. T.: Water load - a now test for prolactın suppression. Clin. Res. 21, 260 (1973).

4. BULBROOK, A. D., WANG. D. Y.: Workshop Human Prolacun. Amsterdam (8/1975).

5. del POZO, E, BRUN del RE, R., VARGA, L., FRIESEN. H. The inhibition of prolactin secretion in man by CB-154. J. clin. Endocr. 35.768 (1972)

6. FLUCKIGER, E.: Regulation der Prolaktin-Sekretion. Gynakologe 10. 62 (1977). 7. HOROBIN. D. F.: Prolactin und fluid and electrolve balance and the cardiovascular system. In: Horobin, D. F.: Prolactin: physiology and clinical significance. Lancaster The Blackburn Times Press (1973).

8. HOROBIN, D. F.: Prolaktin 1975, Eden Press Montreal (1975).

9. HWANG, P.. GUYDA, H., FRIESE, H.: A radioimmunoassay for human prolactin Proc. nat. Acad. Sci. (Wash.) 68, 1902 (1971)

10. JURGENSEN, O., WIRTH, C., vON RAUCH, J., DERICKS-TAN. S. E., von WILD. K.. TAUBERT, H. D.: Bromocriptin (CB.154) in der Behandlung der sekundären Amenorrhoe mit und ohne Hyperprolaktinämie. Nota bene medici 6 (9), 38 (1976) 11. LEIGHTON, P. C., MCNEILLY, A. S., CHARD, T.: Short-term variation in blood levels of prolactin in women. J. Endocr. 68, 177 (1976).

12. LEWIS, U. J., SINGH, R. N. P., SEAVEY, B. K.: Human prolactin: Isolation and some properties. Biochem. biophys. Res. Commun. 44,1169 (1971).

13. LEYENDECKER, G. NOCKE, W., SCHMIDT-GOLLWITZER, M., ENTZIAN, W., del POZO. E.: Klinik der hyperprolaktinämischen Amenorrhœe. Gynäkologe 10, 93 (1977).

14. METTLER, L.: Nomenklatur und Diagnostik der endokrin bedingten weiblichen Sterilität. Fortschritte der Fertilitätsforsch. V, 100, Grosse Berlin 11977).

15. METTLER, L., BRACKEBUSCH, H.-D. SCHMOLCK, A.: Hormonprofile bei Sterilitätspatientinnen unter der Behandlung mit Bromoergokryptin. Arch. of Gynecology, Vol. 228, 588 (1979).

16. SCHMIDT-GOLLWITZER, M., HASAN, S. H., NEVINNY-STIKKEL, Y., FRIEDREICH, E.: Influence of estrogen on prolactin secretion in woman. Acta endocrin. Kbh. Suppl. 193, 30 (1975).

17. SCHMIDT -GOLLWITZER, M., HARDT, W., LEYENDECKER, G.: Hyperprolaktinämie. Diagnostik und Therapie in der Gynäkologie. Gynäkol. Praxis 3, 477 (1979).

18. SCHNEIDER, H.P. G., BOHNET, H. G.: Hyperprolaktinämische Amenorrhoe und Anovulation. Gynäkologe 10, 84 (1977).

19. SCHMOLCK, A., METTLER, L.: Hyper- und Normoprolactinämie bei Amenorrhoe und Galaktorrhoe-Amenorrhoe-Syndrom. Med. Klin. 74, 1273-1278 (36), (1979). 20. SCHULZ, K.-D., GEIGER, W., KÜNZIG, H. J., KLOPPENBURG, W.: Der Einfluß Prolaktin-inhibierender Substanzen auf den normalen Zyklus und auf prolaktinabhāngige, normoprolaktinämische Erkrankungen der Frau.

Gynäkologe 10, 113 (1977).

21. WHO Technical Report Series No. 514 (1976)

\section{Buchbesprechung}

\section{Die Krankheiten der Schilddrüse}

Von K. Oberdisse, E. Klein und D. Reinwein Mit Beiträgen von B. Egloff, Chr. Hedinger, K. Keminger, C. R. Pickardt und P. C. Scriba

\section{2. überarbeitete und erweiterte Auflage}

217 Abbildungen und 324 Einzeldarstellungen, davon 44 farbig, 93 Tabellen

ISBN 3-313-383602-1

Georg Thieme Verlag Stuttgart - New York

Die erste Auflage des Buchs erschien bereits 1967. Nach der Darstellung der Schilddrüsenkrankheiten durch Bansi im Handbuch der Inneren Medizin 1955 war keine umfassende Beschreibung dieses Gebiets mehr erschienen.

Die Erfahrungen der Verfasser, die gemeinsam an der Düsseldorfer Klinik auf diesem Gebiet gearbeitet haben und sehr große Erfahrungen sammeln konnten, waren in dieser ersten Auflage niedergelegt. Sie wurden ergänzt durch einen vorzügliche Darstellung der Weltliteratur und durch Beiträge von Chirurgen und Pathologen, die bei langjähriger Beschäftigung mit.der Schilddrüse und ihren Krankheiten in Österreich und Schweiz besondere Erfahrungen, besonders über den endemischen Kropf, gewinnen konnten.

Bei der Bearbeitung der zweiten Auflage sahen sich die Autoren einem stark angewachsenen Stoff gegenüber, besonders auf dem Gebiet der Grundlagenforschung und der Diagnostik, so daß Teile des Buchs vollständig neu geschrieben werden mußten. Neue Gesichtspunkte haben sich vor allem für die klinische Beurteilung der Hyperthyreose, der Hypothyreose und der Immunothyreoditis ergeben. Methodische Fortschritte auf dem Hormonsektor erlauben ein "Screening" der Schilddrüsenkrankheiten, wie es vorher nicht bekannt war.

Für die meisten diagnostischen Fragen nimmt das Buch feste Standpunkte ein. Diese waren vorher in den Aussprachen der Sektion "Schilddrüse" der Deutschen Gesellschaft für Endokrinologie" diskutiert und übereinstimmend geklärt worden. Trotzdem werden in einigen Teilen auch differente Meinungen vertreten.

Das Buch vermittelt in seiner Ausführlichkeit ein vollständiges detailliertes Wissen über das Gebiet der Schilddrüsenkrankheiten. 